\title{
Lercanidipine attenuates angiotensin II-induced cardiomyocyte hypertrophy by blocking calcineurin-NFAT3 and CaMKII-HDAC4 signaling
}

\author{
YUEZHANG CHEN $^{1}$, JIE YUAN ${ }^{2}$, GUOLIANG JIANG ${ }^{2}$, JIANBING ZHU $^{2}$, YUNZENG ZOU $^{2}$ and QIANZHOU LV ${ }^{1}$ \\ ${ }^{1}$ Department of Pharmacy and ${ }^{2}$ Shanghai Institute of Cardiovascular Diseases, \\ Zhongshan Hospital, Fudan University, Shanghai 200032, P.R. China
}

Received October 27, 2016; Accepted June 19, 2017

DOI: 10.3892/mmr.2017.7211

\begin{abstract}
Previous studies have demonstrated that lercanidipine, a calcium channel blocker, may protect against cardiac hypertrophy; however, the underlying mechanisms remain unclear. In the present study, the effects of lercanidipine on hypertrophy and the mechanisms involved were investigated. Cardiomyocytes isolated from neonatal rats were cultured and treated with angiotensin II (Ang II) in the presence or absence of lercanidipine or tacrolimus (FK506, a calcineurin inhibitor). Reverse transcription-quantitative polymerase chain reaction was used to assess the mRNA expression of genes of interest, whereas the protein expression of calcium-dependent signaling molecules was detected using western blot analysis. In addition, the cell surface area and the nuclear translocation of target proteins were evaluated using immunofluorescence. The results of the present study demonstrated that lercanidipine and FK506 inhibited Ang II-induced cardiomyocyte hypertrophy, as evidenced by decreases in fetal gene (atrial natriuretic peptide and brain natriuretic peptide) expression levels and cell surface area. Notably, lercanidipine suppressed Ang II-induced activation of calcineurin A (CnA) and nuclear factor of activated T cells 3 (NFAT3). In addition, calcium/calmodulin-dependent kinase II (CaMKII)-histone deacetylase 4 (HDAC4) signaling was also inhibited by lercanidipine. In conclusion, the present study demonstrated that lercanidipine may ameliorate cardiomyocyte
\end{abstract}

Correspondence to: Professor Qianzhou Lv, Department of Pharmacy, Zhongshan Hospital, Fudan University, 180 Fenglin Road, Shanghai 200032, P.R. China

E-mail: 1v.qianzhou@zs-hospital.sh.cn

Professor Yunzeng Zou, Shanghai Institute of Cardiovascular Diseases, Zhongshan Hospital, Fudan University, 180 Fenglin Road, Shanghai 200032, P.R. China

E-mail: yunzengzou1024@aliyun.com

Key words: cardiomyocyte hypertrophy, lercanidipine, FK506, calcineurin, nuclear factor of activated $\mathrm{T}$ cells 3 , calcium/calmodulin-dependent kinase II, histone deacetylase 4 hypertrophy, possibly partially by blocking Cn-NFAT3 and CaMKII-HDAC4 signaling.

\section{Introduction}

Cardiac hypertrophy is considered a compensatory mechanism of the heart that maintains cardiac output during various physiological and pathological conditions, including hypertension. However, sustained hypertrophy often leads to decompensation, resulting in heart failure and sudden death $(1,2)$. At the single cardiomyocyte level, hypertrophy is characterized by an increase in cardiomyocyte size and the activation of fetal genes, such as atrial natriuretic peptide (ANP) and brain natriuretic peptide (BNP), and is regulated by numerous signaling cascades. Among the signaling cascades implicated in cardiac hypertrophy, two calcium-dependent pathways are particularly important: Calcineurin (Cn)-nuclear factor of activated T cells 3 (NFAT3) and calcium-calmodulin (CaM)-dependent kinase II (CaMKII)-histone deacetylase 4 (HDAC4) (3-5).

$\mathrm{Cn}$, which is also known as protein phosphatase $2 \mathrm{~B}$, is composed of a catalytic $\mathrm{CnA}$ subunit and a regulatory $\mathrm{CnB}$ subunit, and is a CaM-dependent serine/threonine phosphatase. Once activated by sustained elevations of intracellular calcium, Cn dephosphorylates NFAT3, enabling its translocation to the nucleus, with consequent activation of prohypertrophic target genes (6). Similarly, CaMKII is known to phosphorylate HDAC4, promoting the shuttling of HDAC4 from the nucleus to the cytoplasm, thereby activating prohypertrophic target genes $(7,8)$.

Compared with these prohypertrophic pathways, several classes of antihypertensive drugs, including angiotension converting enzyme inhibitor (ACEI), angiotensin II receptor blocker (ARB) and calcium channel blocker (CCB), have been demonstrated to reverse hypertrophy in humans $(9,10)$. CCB induces regression of hypertrophy to a similar extent to ACEI/ARB (9,10). Furthermore, CCB may be an alternative to ACEI/ARB, if patients are intolerant to ACEI/ARB or if these drugs are contraindicated.

In this context, lercanidipine, which is a third-generation L-type dihydropyridine (DHP) CCB, was of interest in the present study. It is highly lipophilic and is stored within cell membranes, hence its slow onset and long duration of 
action (11). Taken once per day, lercanidipine induces even and sustained blood pressure reduction over $24 \mathrm{~h}$ (11). Compared with other DHP CCBs, lercanidipine has an equivalent antihypertensive efficacy and is better tolerated (12).

In vivo studies revealed that lercanidipine blocks hypertrophy $(13,14)$; however, the underlying mechanism remains unclear. Since lercanidipine is an L-type CCB, and L-type calcium channels are considered the primary source of calcium that activates Cn-NFAT3 signaling (15), it was speculated that lercanidipine may inhibit Cn-NFAT3 and CaMKII-HDAC4 signaling, which may be associated with the antihypertrophic effects of lercanidipine. The primary aim of the present study was to investigate whether lercanidipine induced the regression of cardiomyocyte hypertrophy, and whether lercanidipine inhibited Cn-NFAT3 and CaMKII-HDAC4 signaling. To generate a hypertrophy model, neonatal rat ventricular myocytes (NRVMs) were stimulated with angiotensin II (Ang II). In addition, the effects of tacrolimus (FK506), which is a pharmacological inhibitor of $\mathrm{Cn}$, on hypertrophy, Cn-NFAT3 and CaMKII-HDAC4 signaling were investigated.

\section{Materials and methods}

Reagents. Human Ang II (cat. no. 641206) was purchased from Wako Pure Chemical Industries, Ltd. (Osaka, Japan). FK506 (cat. no. S5003) was purchased from Selleck Chemicals (Houston, TX, USA). Lercanidipine hydrochloride (cat. no. L6668) was purchased from Sigma-Aldrich; Merck KGaA (Darmstadt, Germany). Anti-CnA antibody (1:10,000, cat. no. ab52761; rabbit monoclonal antibody) and anti-CaMKII antibody (1:2,000, cat. no. ab52476; rabbit monoclonal antibody) were purchased from Abcam (Cambridge, UK). Anti-NFAT3 antibody (for western blotting, 1:1,000, cat. no. 2183; rabbit monoclonal antibody) and anti-HDAC4 antibody $(1: 1,000$, cat. no. 7628; rabbit monoclonal antibody) were purchased from Cell Signaling Technology, Inc. (Danvers, MA, USA). Anti-NFAT3 antibody (for immunofluorescence assay, 1:200, cat. no. sc-13036; rabbit polyclonal antibody) was purchased from Santa Cruz Biotechnology, Inc. (Dallas, TX, USA).

Cell culture and treatment. Experiments were performed in compliance with the Guide for the Care and Use of Laboratory Animals published by the National Institutes of Health (publication no. 85-23; Bethesda, MD, USA) and were approved by the Animal Care Committee of Zhongshan Hospital, Fudan University (Shanghai, China). Sprague-Dawley rats (age, 1-3 days; weight, 6-10 g; $n=88$ ) were purchased from the Center of Experimental Animals of Fudan University (Shanghai, China). Rats were maintained under controlled housing conditions (temperature, $22-26^{\circ} \mathrm{C}$; humidity, 40-60\%) with a 12-h light/dark cycle. Water and food were available ad libitum. Following ether inhalation anesthesia, rats were sacrificed by decapitation. Then, hearts were obtained under sterile conditions, washed with PBS twice and were thoroughly minced with scissors. Tissue was digested with $0.1 \%$ trypsin in PBS at $37^{\circ} \mathrm{C}$ with agitation. In total, tissues were digested 6-8 times, and each digestion persisted for $8 \mathrm{~min}$. After each digestion, $10 \mathrm{ml}$ supernatant was carefully collected (the first supernatant was discarded), $10 \mathrm{ml}$ fresh $0.1 \%$ trypsin was added to the buffer and the next digestion was started. Digestion in the supernatant was terminated with isopyknic $10 \%$ fetal bovine serum (FBS; Invitrogen; Thermo Fisher Scientific, Inc., Waltham, MA, USA) in PBS and pelleted by centrifugation at $1,200 \mathrm{x}$ g for $6 \mathrm{~min}$ at room temperature. The pelleted cells were resuspended in Dulbecco's modified Eagle medium (Invitrogen; Thermo Fisher Scientific, Inc.) containing $10 \% \mathrm{FBS}$ and $1 \%$ penicillin-streptomycin. After digestion and resuspension, the cell suspension was filtered through a 400-mesh filter and cultured for $120 \mathrm{~min}$ at $37^{\circ} \mathrm{C}$. The supernatant was seeded in plates at a density of $1 \times 10^{6}$ cells/35-mm plate. After $24 \mathrm{~h}$ serum starvation NRVMs were treated with $2 \times 10^{-7} \mathrm{M}$ Ang II for $12 \mathrm{~h}$ (RNA-associated experiments) or $24 \mathrm{~h}$ (protein-associated experiments). FK506 $\left(10^{-7} \mathrm{M}\right)$ or lercanidipine $\left(10^{-7}, 10^{-6}\right.$ or $\left.10^{-5} \mathrm{M}\right)$ was added to the culture medium $1 \mathrm{~h}$ prior to Ang II administration. The temperature of incubation was $37^{\circ} \mathrm{C}$. Untreated cells served as the control groups.

Reverse transcription-quantitative polymerase chain reaction $(R T-q P C R)$. Total RNA was extracted from cells using TRIzol (Invitrogen; Thermo Fisher Scientific, Inc.), and cDNA was synthesized using a FastQuant RT kit with gDNase (Tiangen Biotech Co., Ltd., Beijing, China) according to manufacturer's instructions. PCR was performed using SYBR ${ }^{\circledR}$ Premix Ex Taq (Tli RNaseH Plus; Takara Biotechnology Co., Ltd., Dalian, China). GAPDH was used as an internal control. Cycling conditions were as follows: An initial pre-denaturation step for $10 \mathrm{sec}$ at $95^{\circ} \mathrm{C}$, followed by 45 cycles of denaturation at $95^{\circ} \mathrm{C}$ for $10 \mathrm{sec}$, annealing at $60^{\circ} \mathrm{C}$ for $20 \mathrm{sec}$ and extension at $70^{\circ} \mathrm{C}$ for $30 \mathrm{sec}$, and a final extension step at $70^{\circ} \mathrm{C}$ for $5 \mathrm{~min}$. Quantification was conducted using the comparative $\mathrm{Cq}$ method (16). Primer sequences were as follows: ANP forward, 5'-TCGAGCAGATTTGGCTGTTATCTTC-3' and reverse, 5'-TGACAGGATTGGAGCCCAGAG-3'; BNP forward, 5'-GGGCTGTGACGGGCTGAGGTT-3' and reverse, 5'-AGT TTGTGCTGGAAGATAAGA-3'; and GAPDH forward, 5'-GCATGTCAGATCCACAACGG-3' and reverse, 5'-GCA TGTCAGATCCACAACGG-3'.

Western blotting. Cells were lysed in a buffer ( $\mathrm{pH} 7.4)$ containing $1 \%$ Nonidet P-40, $1 \%$ deoxycholate, $0.1 \%$ sodium dodecyl sulfate, $150 \mathrm{mmol} / \mathrm{l}$ sodium chloride and $10 \mathrm{mmol} / \mathrm{l}$ Tris-HCl. Subsequently, cell debris was removed by centrifugation at $12,000 \times \mathrm{g}$ for $30 \mathrm{~min}$ at $4^{\circ} \mathrm{C}$. Protein concentration was measured using a bicinchoninic acid assay kit (Sigma-Aldrich; Merck KGaA). Subsequently, the extracted protein was boiled for $5 \mathrm{~min}$ in $5 \mathrm{X}$ loading buffer. Protein samples $(25 \mu \mathrm{g})$ were separated by $10 \%$ SDS-PAGE and transferred onto polyvinylidene difluoride membranes. Membranes were blocked with 5\% bovine serum albumin (BSA; Sigma-Aldrich; Merck KGaA) for $1 \mathrm{~h}$ at room temperature and were then incubated with primary antibodies at $4^{\circ} \mathrm{C}$ overnight. After 3 washes in TBS containing $0.1 \%$ Tween-20, membranes were incubated with horseradish peroxidase (HRP)-conjugated secondary antibody (1:5,000, cat. no. KC-RB-035; Zhejiang Kangchen Biotech Co., Ltd., Shanghai, China) for $1 \mathrm{~h}$ at room temperature. After 3 washes, antigen-antibody complexes were visualized using Fujifilm LAS-3000 Image Analyzer (Fuji, Tokyo, Japan) and Image Lab version 6.0 software (Bio-Rad Laboratories, 
Inc., Hercules, CA, USA) using a Pro-light HRP chemiluminescent kit (Tiangen Biotech Co.). GAPDH (1:5,000, cat. no. KC-5G5; Zhejiang Kangchen Biotech Co., Ltd.) was used as the internal control. Relative protein levels were determined by densitometry using Image-Pro Plus version 6.0 software (Media Cybernetics, Rockville, MD, USA). After intensity calibration, integrated optical density (IOD) was measured. Relative protein expression levels were semi-quantified as the ratio between the protein of interest IOD and the corresponding GAPDH IOD.

Immunofluorescence assay. For cell surface area measurement, cells $\left(3 \times 10^{5}\right.$ cells/well) were fixed with $4 \%$ paraformaldehyde for $10 \mathrm{~min}$ and permeabilized with $0.2 \%$ Triton $\mathrm{X}-100$ for $5 \mathrm{~min}$. After blocking in 1\% BSA for $1 \mathrm{~h}$, cells were incubated with $2.5 \%$ rhodamine phalloidin for $20 \mathrm{~min}$. Cells were then incubated with $0.02 \%$ DAPI for $5 \mathrm{~min}$. Stained cells were subjected to fluorescence microscopy. All of the above steps were performed at room temperature. Images were analyzed with Image-Pro Plus version 6.0 software. Semi-quantification of cell surface area was performed by measuring 50-random cells from 3 experiments, and the average value was calculated.

For NFAT3 nuclear translocation measurement, cells ( $3 \times 10^{5}$ cells/well) were fixed with $4 \%$ paraformaldehyde for $10 \mathrm{~min}$ and permeabilized with $0.2 \%$ Triton X-100 for $5 \mathrm{~min}$ at room temperature. After being blocked in 5\% normal donkey serum (containing $0.2 \%$ Triton X-100; Jackson ImmunoResearch Laboratories, Inc., West Grove, PA, USA) at room temperature for $1 \mathrm{~h}$, cardiomyocytes were incubated with anti-NFAT3 primary antibody at $4{ }^{\circ} \mathrm{C}$ overnight. Cells were then incubated with goat anti-rabbit immunoglobulin G-fluorescein isothiocyanate secondary antibody (cat. no. sc-2012; Santa Cruz Biotechnology, Inc.) for $1 \mathrm{~h}$ at room temperature. Subsequently, cells were incubated with $0.02 \%$ DAPI for $5 \mathrm{~min}$ at room temperature. Stained cells were then subjected to confocal laser microscopy at room temperature. Images were analyzed with Image-Pro Plus version 6.0 software. Firstly, color images were converted to grey images. After invert contrast, optical density correction and definition of the filter range, IOD and area were measured. The ratio between IOD and the area represented by the NFAT3 fluorescence were measured. Nuclei with NFAT3 fluorescence that was 2-fold above cytoplasmic NFAT3 fluorescence were deemed to be NFAT3-positive. NFAT3 translocation was quantified as the ratio between cells containing NFAT3 localized in their nucleus (NFAT3-positive) and the total number of cells counted.

Statistical analysis. Data are presented as the mean \pm standard error of the mean. Differences between groups were analyzed by one-way analysis of variance followed by Bonferroni multiple comparisons test. $\mathrm{P}<0.05$ was considered to indicate a statistically significant difference.

\section{Results}

Effect of lercanidipine on the expression of ANP/BNP in hypertrophic cardiomyocytes. To investigate whether lercanidipine prevented hypertrophy, an in vitro model of Ang II-induced hypertrophy was used. Since the typical fetal genes ANP
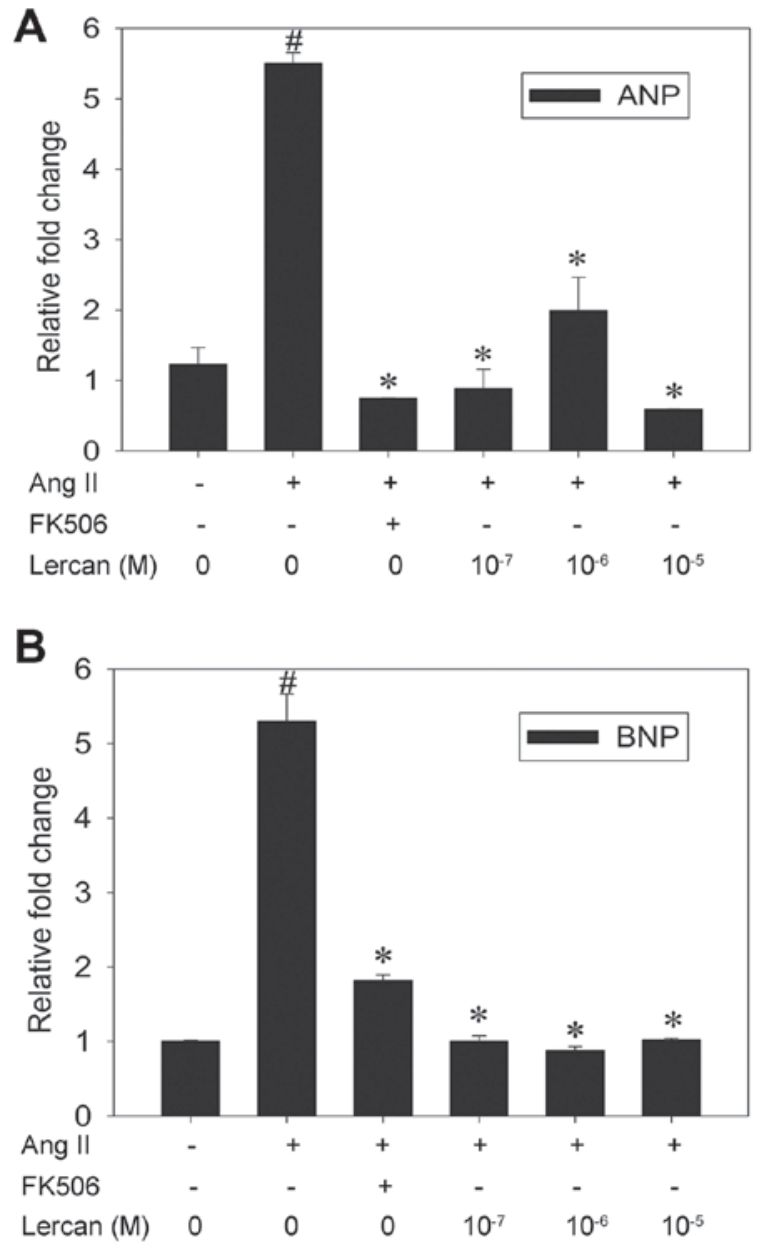

Figure 1. Lercanidipine decreases Ang II-induced elevated ANP and BNP expression in NRVMs. After $24 \mathrm{~h}$ serum starvation, NRVMs were treated with Ang II $\left(2 \times 10^{-7} \mathrm{M}\right)$ for $12 \mathrm{~h}$. FK506 $\left(10^{-7} \mathrm{M}\right)$ or lercanidipine $\left(10^{-7}, 10^{-6}\right.$ or $10^{-5} \mathrm{M}$ ) was added to the culture medium $1 \mathrm{~h}$ prior to Ang II administration. Control cells received no treatment. (A) Quantitative analysis of ANP and (B) BNP expression. Data are presented as the mean \pm standard error of the mean. ${ }^{\#} \mathrm{P}<0.05$ vs. the control group; ${ }^{*} \mathrm{P}<0.05$ vs. the Ang II group. Ang II, angiotensin II; ANP, atrial natriuretic peptide; BNP, brain natriuretic peptide; Lercan, lercanidipine; NRVMs, neonatal rat ventricular myocytes.

and BNP are markers of hypertrophy, the expression of ANP and BNP was determined by RT-qPCR. Results revealed that Ang II $\left(2 \times 10^{-7} \mathrm{M}\right)$ significantly increased the expression levels of ANP (Fig. 1A) and BNP (Fig. 1B) in NRVMs compared with the control group. Pretreatment with FK506 (10 $\left.{ }^{-7} \mathrm{M}\right)$, a well-known inhibitor of $\mathrm{Cn}$, reduced the elevated expression levels of ANP (Fig. 1A) and BNP (Fig. 1B). Similarly, 10 ${ }^{-7}, 10^{-6}$ and $10^{-5} \mathrm{M}$ lercanidipine decreased Ang II-induced elevated expression of ANP (Fig. 1A) and BNP (Fig. 1B). Since various doses of lercanidipine were revealed to significantly decrease the expression levels of ANP and BNP, the median dose $\left(10^{-6} \mathrm{M}\right)$ was selected for use in subsequent experiments.

Effect of lercanidipine on cell surface area in hypertrophic cardiomyocytes. Since an increase in cell surface area is another feature of hypertrophy, an immunofluorescence assay was performed to determine cell surface area. Consistent with the RT-qPCR results, immunofluorescence staining demonstrated that Ang II $\left(2 \times 10^{-7} \mathrm{M}\right)$ effectively induced hypertrophy, as evidenced by an increase in cell surface area (Fig. 2). 


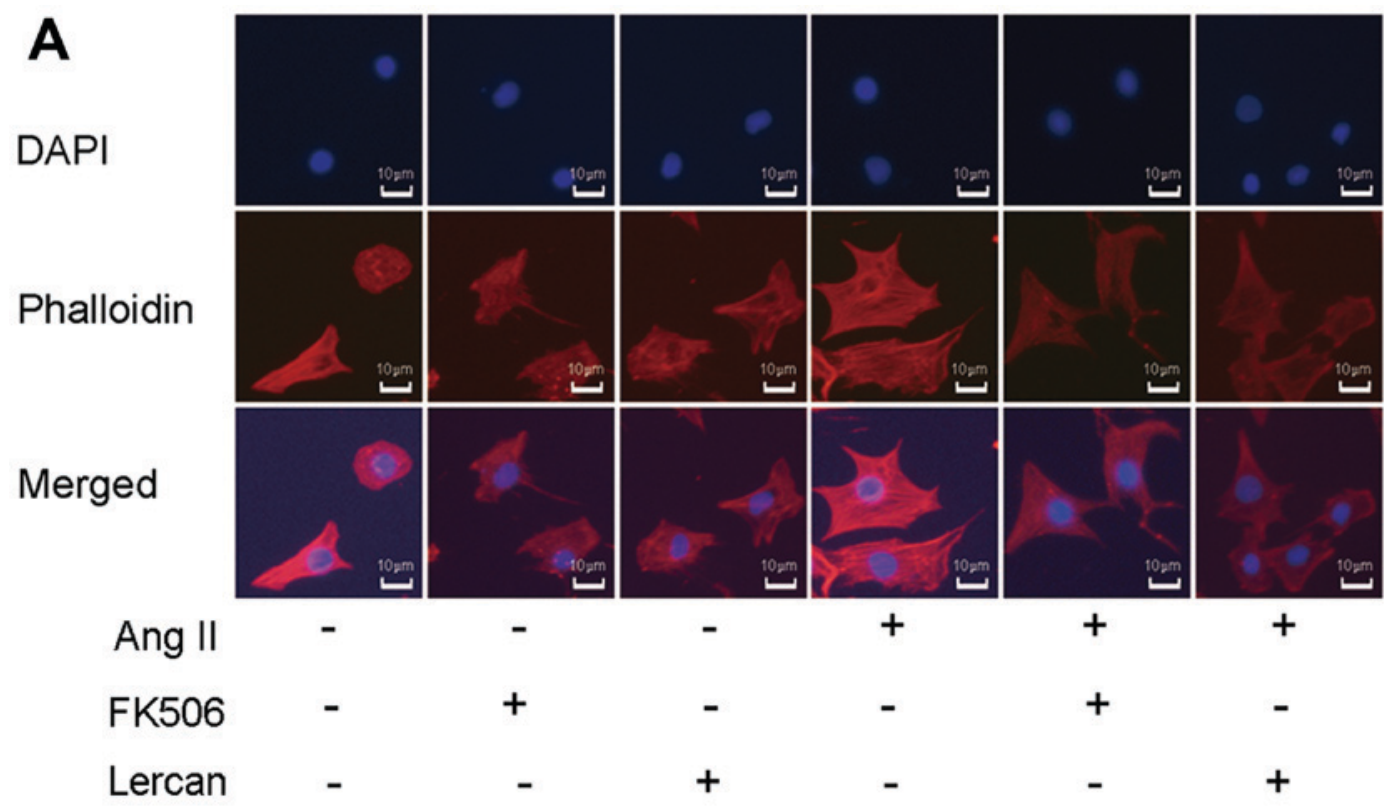

B

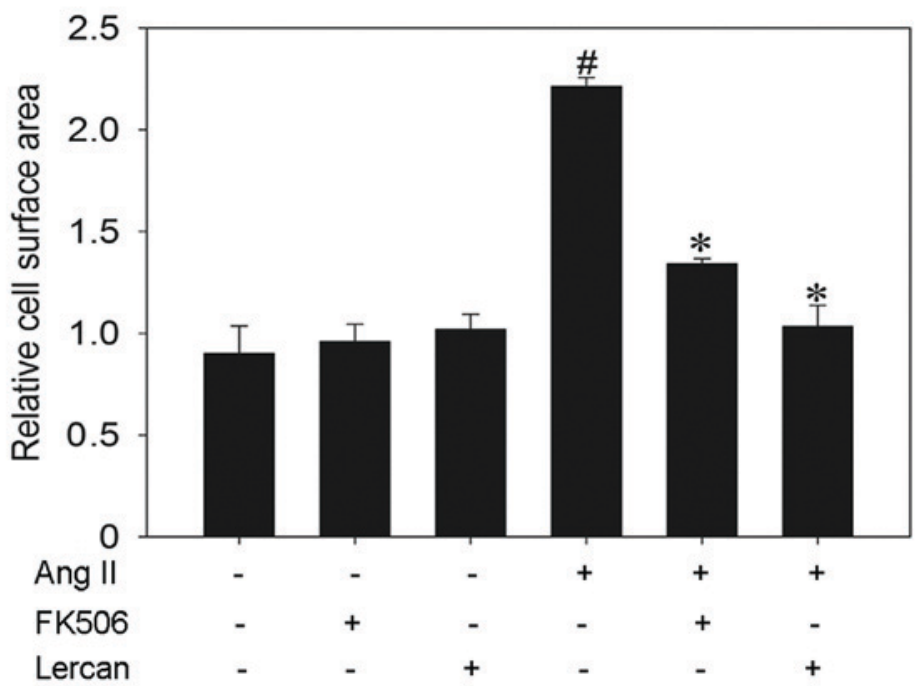

Figure 2. Lercanidipine decreases Ang II-induced cell surface area enlargement in NRVMs. After $24 \mathrm{~h}$ serum starvation, NRVMs were treated with Ang II $\left(2 \times 10^{-7} \mathrm{M}\right)$ for $24 \mathrm{~h}$. FK506 $\left(10^{-7} \mathrm{M}\right)$ or lercanidipine $\left(10^{-6} \mathrm{M}\right)$ was added to the culture medium $1 \mathrm{~h}$ prior to Ang II administration. Control cells received no treatment. (A) Representative images of immunofluorescence staining for phalloidin and DAPI following treatment. (B) Semi-quantitative analysis of cell surface area measurement. Data are presented as the mean \pm standard error of the mean. ${ }^{\#} \mathrm{P}<0.05$ vs. the control group; ${ }^{\mathrm{P}}<0.05$ vs. the Ang II group. Ang II, angiotensin II; Lercan, lercanidipine.

Lercanidipine $\left(10^{-6} \mathrm{M}\right)$ prevented an Ang II-stimulated increase in cell surface area, and the effect was similar in cells treated with $10^{-7} \mathrm{M}$ FK506 (Fig. 2). Taken together, the data in Figs. 1 and 2 suggested that lercanidipine may inhibit the hypertrophic response to Ang II in NRVMs.

Effect of lercanidipine on Cn-NFAT3 and CaMKII-HDAC4 signaling in hypertrophic cardiomyocytes. The signaling mechanisms by which lercanidipine suppressed hypertrophy were also investigated. Since lercanidipine blocks L-type calcium channels, its ability to regulate the expression of proteins associated with two calcium-dependent signaling pathways, Cn-NFAT3 and CaMKII-HDAC4, was analyzed in NRVMs. Exposure of NRVMs to Ang II $\left(2 \times 10^{-7} \mathrm{M}\right)$ resulted in increased expression of $\mathrm{CnA}$ (Fig. 3A and B) and
NFAT3 (Fig. 3A and C). Pretreatment with lercanidipine $\left(10^{-6} \mathrm{M}\right)$ decreased the Ang II-induced elevated expression of CnA (Fig. 3A and B) and NFAT3 (Fig. 3A and C). Treatment with FK506 $\left(10^{-7} \mathrm{M}\right)$ inhibited NFAT3 expression (Fig. 3A and C) without affecting $\mathrm{CnA}$ expression (Fig. 3A and B). In addition, exposure of NRVMs to Ang II led to upregulated expression levels of CaMKII (Fig. 3A and D) and its downstream protein HDAC4 (Fig. 3A and E). Pretreatment with FK506 prevented Ang II-induced CaMKII-HDAC4 signaling activation (Fig. 3A, D and E). Similarly, lercanidipine reduced Ang II-induced upregulation of CaMKII (Fig. 3A and D) and HDAC4 (Fig. 3A and E).

Effect of lercanidipine on NFAT3 nuclear translocation in hypertrophic cardiomyocytes. To confirm that lercanidipine 

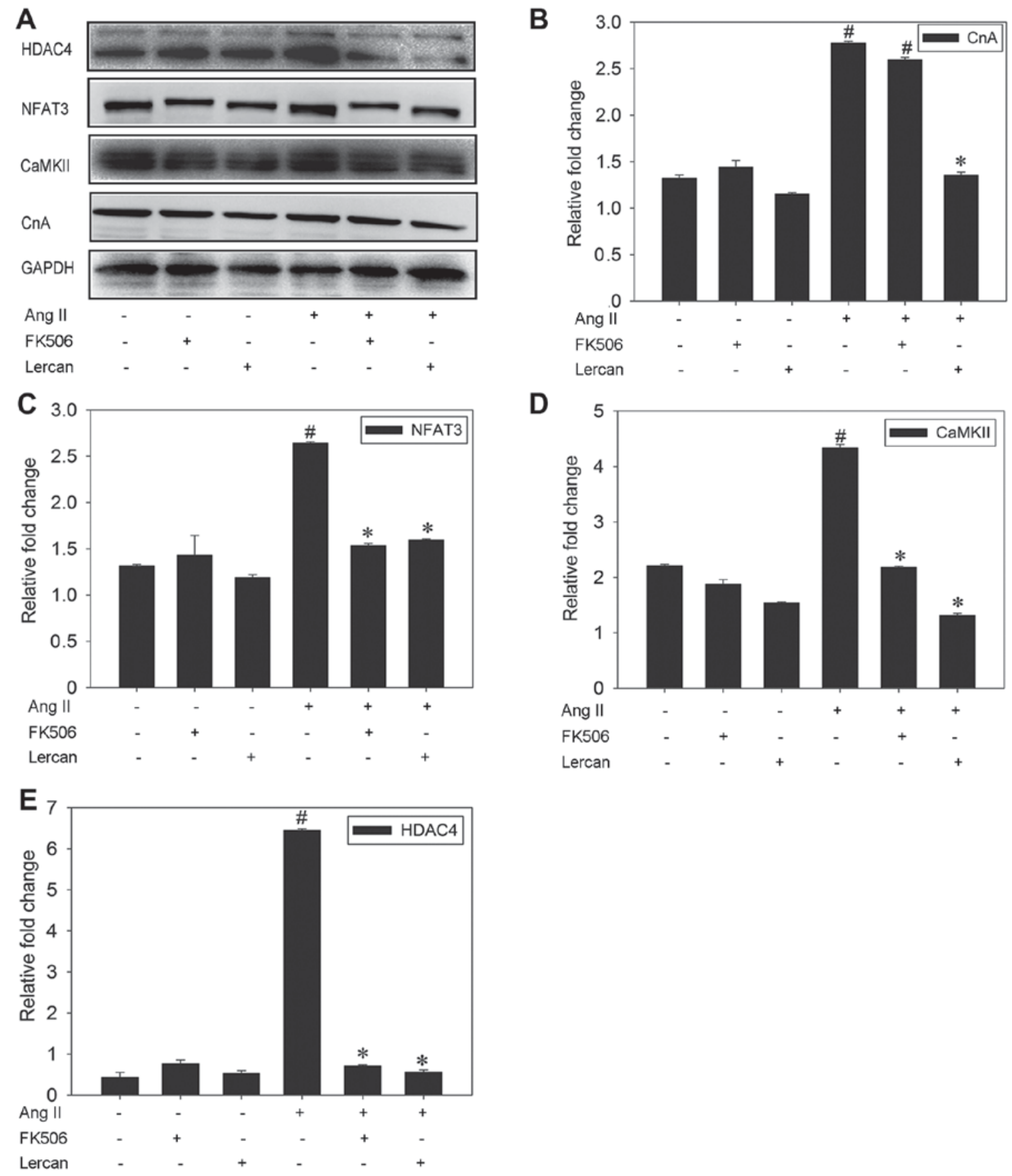

Figure 3. Lercanidipine inhibits Ang II-induced activation of Cn-NFAT3 and CaMKII-HDAC4 signaling in NRVMs. After 24 h serum starvation, NRVMs were treated with Ang II $\left(2 \times 10^{-7} \mathrm{M}\right)$ for $24 \mathrm{~h}$. FK506 $\left(10^{-7} \mathrm{M}\right)$ or lercanidipine $\left(10^{-6} \mathrm{M}\right)$ was added to the culture medium $1 \mathrm{~h}$ prior to Ang II administration. Control cells received no treatment. (A) Representative western blotting images. Densitometric analysis of (B) CnA, (C) NFAT3, (D) CaMKII and (E) HDAC4 expression levels. Data are presented as the mean \pm standard error of the mean. ${ }^{*} \mathrm{P}<0.05$ vs. the control group; ${ }^{*} \mathrm{P}<0.05$ vs. the Ang II group. Ang II, angiotensin II; CaMKII, calcium/calmodulin-dependent kinase II; CnA, calcineurin A; HDAC4, histone deacetylase 4; Lercan, lercanidipine; NFAT3, nuclear factor of activated T cells 3; NRVMs, neonatal rat ventricular myocytes.

attenuated hypertrophy via Cn-NFAT3 signaling, an immunofluorescence assay was performed to detect NFAT3 nuclear translocation. The results demonstrated that NFAT3 underwent translocation to the nucleus in response to Ang II $\left(2 \times 10^{-7} \mathrm{M}\right)$ stimulation (Fig. 4A and B), whereas pretreatment with FK506 (Fig. 4A and B) or lercanidipine (Fig. 4A and B) inhibited Ang II-induced nuclear translocation of NFAT3. The data presented in Figs. 3 and 4 suggested that inhibition of hypertrophy by lercanidipine was mediated, at least in part, by Cn-NFAT3 and CaMKII-HDAC4 signaling.

\section{Discussion}

The present study demonstrated that lercanidipine inhibited Ang II-induced hypertrophy in vitro. Lercanidipine suppressed Ang II-induced activation of CnA, NFAT3, CaMKII and HDAC4, thus suggesting that lercanidipine may attenuate hypertrophy, at least partially, via the Cn-NFAT3 and CaMKII-HDAC4 signaling pathways.

It is well-established that Ang II is a potent inducer of cardiac hypertrophy. In pressure overload-induced cardiac hypertrophy 


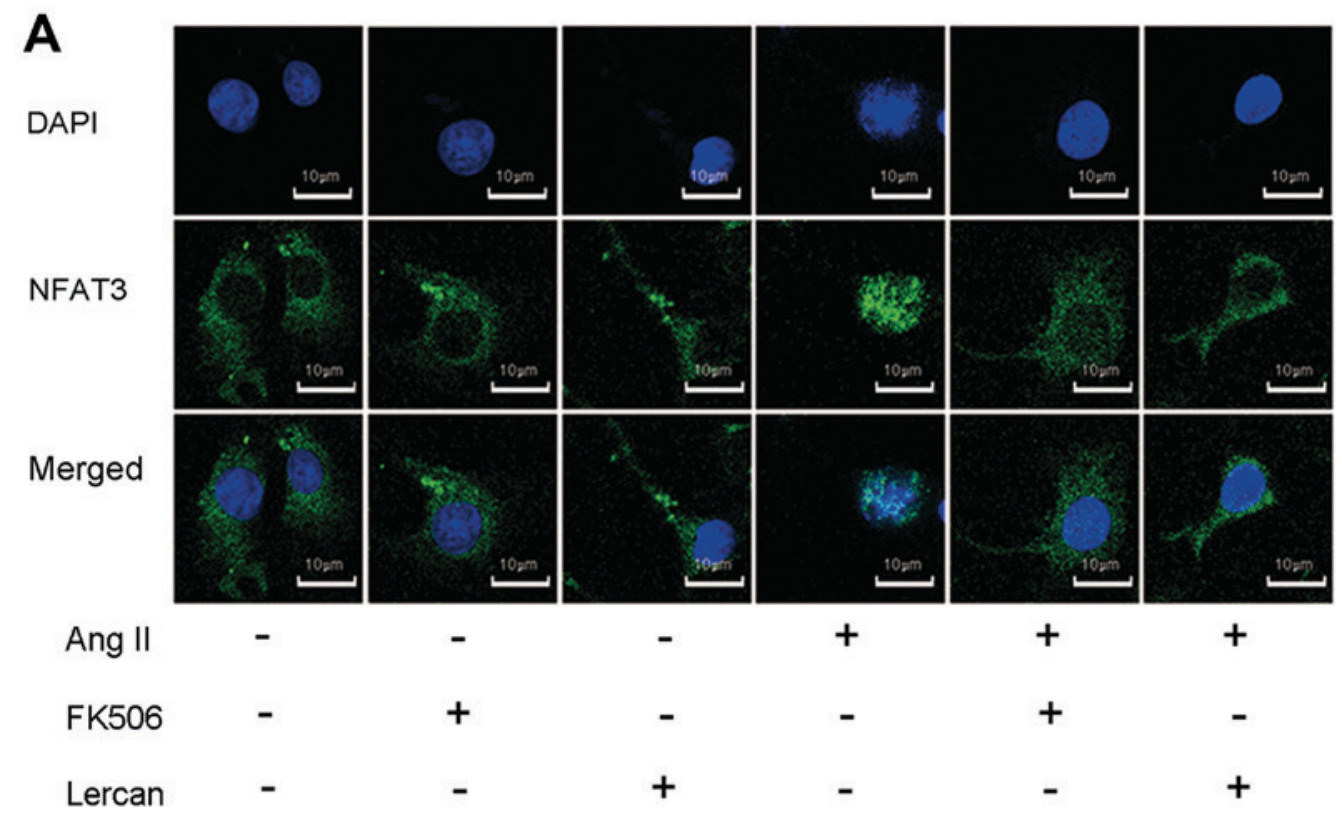

B

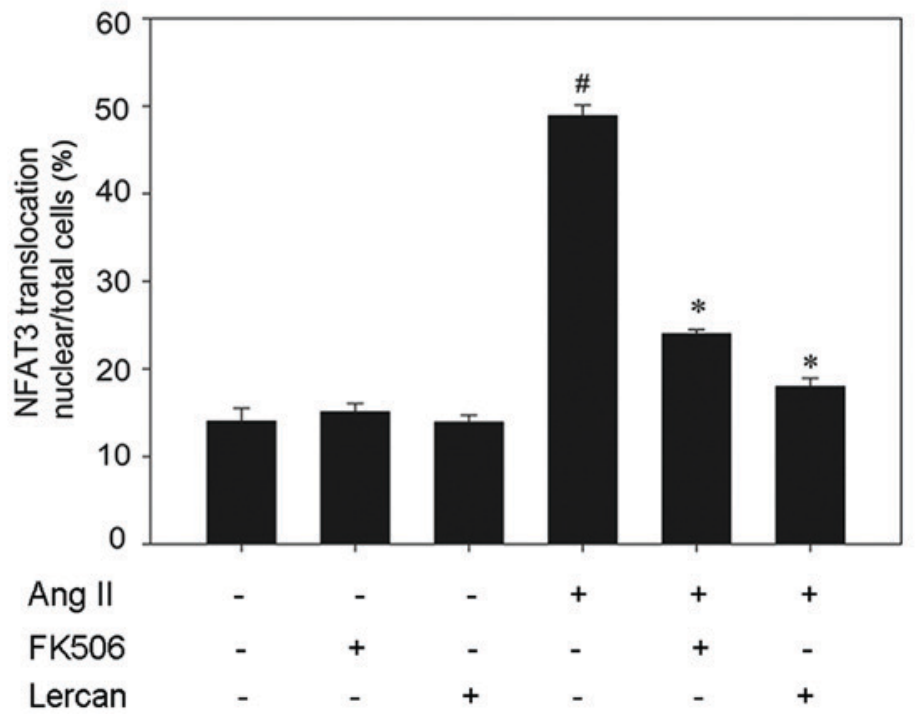

Figure 4. Lercanidipine inhibits Ang II-induced NFAT3 nuclear translocation. After $24 \mathrm{~h}$ serum starvation, NRVMs were treated with Ang II (2x10 $\left.{ }^{-7} \mathrm{M}\right)$ for $24 \mathrm{~h}$. FK506 $\left(10^{-7} \mathrm{M}\right)$ or lercanidipine $\left(10^{-6} \mathrm{M}\right)$ was added to the culture medium $1 \mathrm{~h}$ prior to Ang II administration. Control cells received no treatment. (A) Representative images of immunofluorescence staining of NFAT3. (B) Semi-quantitative analysis of NFAT3 nuclear translocation. Data are presented as the mean \pm standard error of the mean. ${ }^{~} \mathrm{P}<0.05$ vs. the control group; ${ }^{\prime} \mathrm{P}<0.05$ vs. the Ang II group. Ang II, angiotensin II; Lercan, lercanidipine; NFAT3, nuclear factor of activated T cells 3 ; NRVMs, neonatal rat ventricular myocytes.

models, both local cardiac Ang II and circulating Ang II concentrations were increased, and treatment with ACEI/ARB effectively prevented the hypertrophic responses, suggesting that Ang II serves a key role in the development of hypertrophy $(17,18)$. In the present study, it was demonstrated that Ang II induced cardiomyocyte hypertrophy. In addition, the Cn-NFAT3 and CaMKII-HDAC4 signaling pathways were activated in Ang II-induced hypertrophic cardiomyocytes; this finding was concordant with previous studies that suggested an association between Cn-NFAT3 $(6,19)$ and CaMKII-HDAC4 $(7,8,20,21)$ signaling and cardiac hypertrophic responses.

Cyclosporine A (CsA) and FK506 are two calcineurin inhibitors, which are widely used as immunosuppressive agents. The results of the present study revealed that FK506 suppressed Ang II-induced hypertrophy, which was consistent with previous studies that demonstrated that CsA or FK506 attenuated hypertrophy in various models (3). Conversely, certain studies have either observed no effect or a deterioration of hypertrophy with CsA or FK506 $(22,23)$. These discrepancies may be explained by the intrinsic differences between various models. In the present study, administration of FK506 failed to suppress the Ang II-induced increase in CnA expression levels. The reason for this may be that, binding with FK-binding protein 12, FK506 formed a drug-immunophilin complex that sterically suppressed Cn activity (24) without altering the quantity of CnA. Notably, treatment 
with lercanidipine significantly inhibited Ang II-induced cardiomyocyte hypertrophy in the present study, which was concordant with the findings of previous in vivo studies $(13,14)$. In addition, lercanidipine suppressed Ang II-elevated expression of CnA, NFAT3, CaMKII and HDAC4, thus suggesting that lercanidipine attenuated cardiomyocyte hypertrophy by inhibiting the Cn-NFAT3 and CaMKII-HDAC4 signaling pathways. Administration of lercanidipine also prevented Ang II-induced NFAT3 nuclear translocation, which suggested that suppression of Cn-NFAT3 signaling may be associated with the antihypertrophic action of lercanidipine.

Although lercanidipine and FK506 displayed similar inhibitory effects on hypertrophy in the present study, lercanidipine is more efficacious than FK506 for two reasons. Firstly, FK506 is known to cause numerous unwanted side effects, including nephrotoxicity, hypertension and diabetes mellitus, whereas lercanidipine is generally well tolerated in patients with hypertension. Specifically, lercanidipine exerts slighter side effects in the heart (negative inotropism and reflex tachycardia) when compared with other CCBs, such as nifedipine and amlodipine (25-31). Secondly, lercanidipine may exert pleiotropic roles that go beyond the reduction of blood pressure. It not only has a cardioprotective role, but also serves antiatherogenic $(32,33)$, nephroprotective $(34,35)$ and neuroprotective $(36,37)$ roles. Therefore, due to its numerous therapeutic benefits, lercanidipine may be considered a promising choice for the treatment of patients with cardiac hypertrophy.

In conclusion, the present study demonstrated that lercanidipine attenuates Ang II-induced cardiomyocyte hypertrophy, which may be partially associated with downregulation of the Cn-NFAT3 and CaMKII-HDAC4 signaling pathways. These findings may aid the understanding of the mechanisms underlying the beneficial effects of lercanidipine on hypertrophy, and may provide evidence for the application of lercanidipine in the treatment of hypertrophy.

\section{Acknowledgements}

The present study was supported by the Fund for New One Hundred People Project from Shanghai Municipal Commission of Health and Family Planning. The authors would like to thank Guoping Zhang (Institutes of Biomedical Sciences, Fudan University, Shanghai, China) for his technical support and Dr. Yuezhen Dai (College of Foreign Languages and Literatures, Fudan University, Shanghai, China) for her language guidance.

\section{References}

1. Brown DW, Giles WH and Croft JB: Left ventricular hypertrophy as a predictor of coronary heart disease mortality and the effect of hypertension. Am Heart J 140: 848-856, 2000.

2. de Simone G, Gottdiener JS, Chinali M and Maurer MS: Left ventricular mass predicts heart failure not related to previous myocardial infarction: The Cardiovascular Health Study. Eur Heart J 29: 741-747, 2008.

3. Wilkins BJ and Molkentin JD: Calcium-calcineurin signaling in the regulation of cardiac hypertrophy. Biochem Biophys Res Commun 322: 1178-1191, 2004.

4. Tham YK, Bernardo BC, Ooi JY, Weeks KL and McMullen JR: Pathophysiology of cardiac hypertrophy and heart failure: Signaling pathways and novel therapeutic targets. Arch Toxicol 89: 1401-1438, 2015.
5. Anderson ME, Brown JH and Bers DM: CaMKII in myocardial hypertrophy and heart failure. J Mol Cell Cardiol 51: 468-473, 2011.

6. Molkentin JD, Lu JR, Antos CL, Markham B, Richardson J, Robbins J, Grant SR and Olson EN: A calcineurin-dependent transcriptional pathway for cardiac hypertrophy. Cell 93: 215-228, 1998.

7. Backs J, Song K, Bezprozvannaya S, Chang S and Olson EN: CaM kinase II selectively signals to histone deacetylase 4 during cardiomyocyte hypertrophy. J Clin Invest 116: 1853-1864, 2006.

8. Zhang T, Kohlhaas M, Backs J, Mishra S, Phillips W, Dybkova N, Chang S, Ling H, Bers DM, Maier LS, et al: CaMKIIdelta isoforms differentially affect calcium handling but similarly regulate HDAC/MEF2 transcriptional responses. J Biol Chem 282: 35078-35087, 2007.

9. Klingbeil AU, Schneider M, Martus P, Messerli FH and Schmieder RE: A meta-analysis of the effects of treatment on left ventricular mass in essential hypertension. Am J Med 115: 41-46, 2003.

10. Fagard RH, Celis H, Thijs L and Wouters S: Regression of left ventricular mass by antihypertensive treatment: A meta-analysis of randomized comparative studies. Hypertension 54: 1084-1091, 2009.

11. Herbette LG, Vecchiarelli M, Sartani A and Leonardi A: Lercanidipine: Short plasma half-life, long duration of action and high cholesterol tolerance. Updated molecular model to rationalize its pharmacokinetic properties. Blood Press Suppl 2: 10-17, 1998.

12. Borghi C: Lercanidipine in hypertension. Vasc Health Risk Manag 1: 173-182, 2005.

13. Tomassoni D, Sabbatini M and Amenta F: Effect of different dihydropyridine-type $\mathrm{Ca}^{2+}$ antagonists on left ventricle hypertrophy and coronary changes in spontaneously hypertensive rats. J Cardiovasc Pharmacol 41: 544-552, 2003.

14. Amenta F, Peleg E, Tomassoni D, Sabbatini M and Rosenthal T: Effect of treatment with lercanidipine on heart of Cohen-Rosenthal diabetic hypertensive rats. Hypertension 41: 1330-1335, 2003.

15. Gao H, Wang F, Wang W, Makarewich CA, Zhang H, Kubo H, Berretta RM, Barr LA, Molkentin JD and Houser SR: Ca(2+) influx through L-type $\mathrm{Ca}(2+)$ channels and transient receptor potential channels activates pathological hypertrophy signaling. J Mol Cell Cardiol 53: 657-667, 2012.

16. Livak KJ and Schmittgen TD: Analysis of relative gene expression data using real-time quantitative PCR and the 2(-Delta Delta C(T)) Method. Methods 25: 402-408, 2001.

17. Yamamoto $K$, Ohishi $M$, Katsuya $T$, Ito N, Ikushima $M$, Kaibe M, Tatara Y, Shiota A, Sugano S, Takeda S, et al: Deletion of angiotensin-converting enzyme 2 accelerates pressure overload-induced cardiac dysfunction by increasing local angiotensin II. Hypertension 47: 718-726, 2006.

18. Baker KM, Chernin MI, Wixson SK and Aceto JF: Renin-angiotensin system involvement in pressure-overload cardiac hypertrophy in rats. Am J Physiol 259: H324-H332, 1990.

19. Haq S, Choukroun G, Lim H, Tymitz KM, del Monte F, Gwathmey J, Grazette L, Michael A, Hajjar R, Force T and Molkentin JD: Differential activation of signal transduction pathways in human hearts with hypertrophy versus advanced heart failure. Circulation 103: 670-677, 2001.

20. Zhang T, Maier LS, Dalton ND, Miyamoto S, Ross J Jr, Bers DM and Brown JH: The deltaC isoform of CaMKII is activated in cardiac hypertrophy and induces dilated cardiomyopathy and heart failure. Circ Res 92: 912-919, 2003.

21. Backs J, Backs T, Neef S, Kreusser MM, Lehmann LH, Patrick DM, Grueter CE, Qi X, Richardson JA, Hill JA, et al: The delta isoform of $\mathrm{CaM}$ kinase II is required for pathological cardiac hypertrophy and remodeling after pressure overload. Proc Natl Acad Sci 106: 2342-2347, 2009.

22. Hayashida W, Kihara Y, Yasaka A and Sasayama S: Cardiac calcineurin during transition from hypertrophy to heart failure in rats. Biochem Biophys Res Commun 273: 347-351, 2000.

23. Zhang W, Kowal RC, Rusnak F, Sikkink RA, Olson EN and Victor RG: Failure of calcineurin inhibitors to prevent pressure-overload left ventricular hypertrophy in rats. Circ Res 84 : 722-728, 1999.

24. Liu J, Farmer JD Jr, Lane WS, Friedman J, Weissman I and Schreiber SL: Calcineurin is a common target of cyclophilin-cyclosporin A and FKBP-FK506 complexes. Cell 66: 807-815, 1991.

25. AngelicoP,GuarneriL,Leonardi A and Testa R:Vascular-selective effect of lercanidipine and other 1,4-dihydropyridines in isolated rabbit tissues. J Pharm Pharmacol 51: 709-714, 1999. 
26. Guarneri L, Angelico P, Ibba M, Poggesi E, Taddei C, Leonardi A and Testa R: Pharmacological in vitro studies of the new 1,4-dihydropyridine calcium antagonist lercanidipine. Arzneimittelforschung 46: 15-24, 1996.

27. Brixius K, Gross T, Tossios P, Geissler HJ, Mehlhorn U, Schwinger RH and Hekmat K: Increased vascular selectivity and prolonged pharmacological efficacy of the L-type $\mathrm{Ca}^{2+}$ channel antagonist lercanidipine in human cardiovascular tissue. Clin Exp Pharmacol Physiol 32: 708-713, 2005.

28. Omboni S and Zanchetti A: Antihypertensive efficacy of lercanidipine at $2.5,5$ and $10 \mathrm{mg}$ in mild to moderate essential hypertensives assessed by clinic and ambulatory blood pressure measurements. Multicenter Study Investigators. J Hypertens 16 : 1831-1838, 1998.

29. Barbagallo M and Barbagallo Sangiorgi G: Efficacy and tolerability of lercanidipine in monotherapy in elderly patients with isolated systolic hypertension. Aging (Milano) 12:375-379, 2000

30. Fogari R, Mugellini A, Zoppi A, Corradi L, Rinaldi A, Derosa G and Preti P: Differential effects of lercanidipine and nifedipine GITS on plasma norepinephrine in chronic treatment of hypertension. Am J Hypertens 16: 596-599, 2003.

31. Grassi G, Seravalle G, Turri C, Bolla G and Mancia G: Short-versus long-term effects of different dihydropyridines on sympathetic and baroreflex function in hypertension. Hypertension 41: 558-562, 2003.
32. Rachmani R, Levi Z, Zadok BS and Ravid M: Losartan and lercanidipine attenuate low-density lipoprotein oxidation in patients with hypertension and type 2 diabetes mellitus: A randomized, prospective crossover study. Clin Pharmacol Ther 72: 302-307, 2002.

33. Yeh JL, Hsu JH, Liang JC, Chen IJ and Liou SF: Lercanidipine and labedipinedilol-A attenuate lipopolysaccharide/interferon- $\gamma$-induced inflammation in rat vascular smooth muscle cells through inhibition of HMGB1 release and MMP-2, 9 activities. Atherosclerosis 226: 364-372, 2013.

34. Sabbatini M, Leonardi A, Testa R, Vitaioli L and Amenta F: Effect of calcium antagonists on glomerular arterioles in spontaneously hypertensive rats. Hypertension 35: 775-779, 2000.

35. Sabbatini M, Leonardi A, Testa R, Tomassoni D, Vitaioli L and Amenta F: Effects of dihydropyridine-type $\mathrm{Ca} 2+$ antagonists on the renal arterial tree in spontaneously hypertensive rats. J Cardiovasc Pharmacol 39: 39-48, 2002.

36. Gupta S, Sharma U, Jagannathan NR and Gupta YK: Neuroprotective effect of lercanidipine in middle cerebral artery occlusion model of stroke in rats. Exp Neurol 288: 25-37, 2016.

37. Sakurai-Yamashita Y, Harada N and Niwa M: Lercanidipine rescues hippocampus pyramidal neurons from mild ischemia-induced delayed neuronal death in SHRSP. Cell Mol Neurobiol 31: 561-567, 2011. 